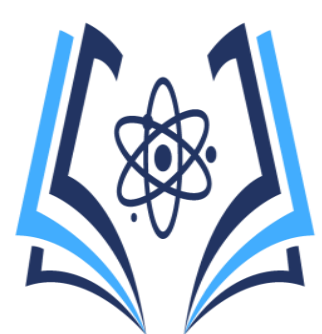

\title{
El proceso de atención de enfermería en pacientes con diabetes mellitus
}

\author{
The nursing care process in patients with diabetes mellitus
}

\section{El proceso de atención de enfermería}

Lcda. Dolores Mirella Cedeño Holguín

Ing. Maribel Celi Vásquez Paucar ${ }^{2}$

Universidad Estatal del Sur de Manabí; e-mail doloresmirella@hotmail.com; dolores.cedeno@unesum.edu.ec, Codigo Orcid: https://orcid.org/0000-0002-7857-9396

\section{Contacto: doloresmirella@hotmail.com}

\section{Recibido: 15-10-2019}

Aprobado: 10-12-2019

\section{Resumen}

El presente trabajo se desarrolló con el objetivo de estudiar el papel de los cuidados de enfermería en pacientes con diabetes mellitus para el conocimiento de los factores de riesgo asociados a esta enfermedad, el problema a estudiar se basó en que, la diabetes mellitus es un malestar crónicadegenerativa que afecta al ser humano, la mayoría de pacientes no suelen detectar la enfermedad a tiempo lo que conlleva a largo plazo a presentar malestar, ausentarse del trabajo, incapacidad, limitaciones, años perdidos de vida, es la principal causante de morbilidad, mortalidad y discapacidad, ha incrementado notablemente en los últimos tiempos alcanzando altas cifras epidémicas, por lo que es de suma importancia prevenirla para evitar que siga incrementando sus estadísticas a nivel mundial. El documento científico se desplegó con la metodología de corte bibliográfico, en el que se analizó material científico relevante que aportó con información necesaria para la investigación, proporcionando al investigador pautas para aplicar y desplegar el control y mejora de la diabetes mellitus en pacientes diagnosticados con esta padecimiento. El resultado del proyecto fue adquisición acreditada y comprobada de nuevos conocimientos sobre la Diabetes mellitus, procesos de atención de enfermería y plan de cuidados, con los sapiencias alcanzadas se presenta una propuesta de plan de cuidados para pacientes que sufren esta patología.

Palabras clave: Intervenciones, personal, evolución, información, patología, riesgos.

\footnotetext{
Abstract

The present work was developed with the objective of studying the role of nursing care in patients with diabetes mellitus for the knowledge of the risk factors associated with this disease, the problem to be studied was based on the fact that diabetes mellitus is a discomfort chronic-degenerative that affects humans, most patients do not usually detect
}

the disease in time, which in the long term leads to discomfort, absence from work, disability, limitations, lost years of life, it is the main cause of morbidity, Mortality and disability, has increased notably in recent times reaching high epidemic figures, so it is extremely important to prevent it to prevent it from continuing to increase its statistics worldwide. The scientific document was deployed with the bibliographic cut methodology, in which relevant scientific material was analyzed that provided the necessary information for the research, providing the researcher with guidelines to apply and deploy the control and improvement of diabetes mellitus in patients diagnosed with it. Suffering. The result of the project was accredited and proven acquisition of new knowledge about Diabetes mellitus, nursing care processes and care plan, with the knowledge achieved, a proposal for a care plan for patients suffering from this pathology is presented.

Keywords: Interventions, personal, evolution, information, pathology, risks. 


\section{Introducción}

De acuerdo a información de la Organización Mundial de la Salud (OMS), en el mundo, más de 425 millones de personas viven actualmente con diabetes y esta enfermedad en 2017 causó 4 millones de muertes. Mientras que, en el Ecuador, de acuerdo a los registros del MSP, hasta junio de 2018 se realizaron 34.597 nuevas atenciones con diagnóstico de diabetes mellitus de las cuales el 98,18\% corresponde a diabetes mellitus tipo 2. Con este contexto y en aras de brindar atención al paciente, el personal de enfermería, aplica procesos de Atención, lo que en el estudio del método científico se identifica como el procedimiento asistencial, el cual permite a los profesionales brindar los cuidados que requiere el paciente, la familia y la sociedad de una forma estructurada, racional y sistemática. Es muy importante desarrollar un plan de cuidados en pacientes con diabetes mellitus para no llegar a tener complicaciones en su salud, instruyéndoles a llevar una vida saludable acompañada de conocimientos brindados por el personal para ejecutar su plan de cuidado con resultados positivos, enfocándose cinco estrategias: identificación del riesgos, trabajo interdisciplinar, estrategias preventivas prácticas, capacitación continua y supervisión. (Cynthia Colttersa, 2019)

Características del proceso de atención de enfermería.

El proceso de atención de enfermería se caracteriza por ser:

Sistemático. - Consta de cinco fases los cuales se encaminan a una serie de acciones deliberadas para alcanzar los resultados establecidos.

Dinámico. - Se adquiera más destreza, podrá desplazarse hacia atrás y hacia delante.

Humanístico. - Cabe destacar que los cuidados se planifican y se ejecutan teniendo en cuenta las necesidades, intereses, valores y deseos específicos del paciente.

Etapas del proceso de atención de enfermería.

Valoración: Es un procedimiento planificado, sistemático, continúo y deliberado de recolección e interpretación de información de la situación de salud que están viviendo las personas y la solución a esta. (Enfermería, 2018)

Se lleva a cabo mediante 3 acciones que son fundamentales: Observación, exploración y entrevista.

La validación de los datos permite al profesional de enfermería realizar los siguientes pasos:

- Asegurarse de que la información recolectada durante la valoración sea completa.

- Garantizar de que los datos objetivos y subjetivos relacionados concuerdan.
- Conseguir información adicional que pueda haberse pasado por alto.

Diagnóstico: NANDA lo define como "juicio clínico sobre las experiencias/respuestas de una persona, familia, grupo o comunidad frente a problemas de salud o procesos vitales reales $o$ potenciales y proporciona la base para la selección de las intervenciones de enfermería destinadas a lograr los resultados de los que la enfermera es responsable".

Planeación: En ella se establece el plan de cuidado mediante la proyeccion y diseño de intervenciones generadas de los diagnosticos planteados.Se definen $\mathrm{y}$ validan intervenciones seguras, pertinentes $\mathrm{y}$ priorizadas, que optimicen sus acciones.

En esta etapa se establecen: Prioridades en la atencion en salud, objetivos para el uso racional de medicamentos, intervenciones de enfermeria y seguimiento y registro del plan de cuidado en la historia clinica.

Ejecución: En esta fase se realizarán todas las intervenciones enfermeras dirigidas a la resolución de problemas y las necesidades asistenciales de cada persona tratada. Es un elemento con mayor importancia que debe realizar el profesional de enfermería gracias a ello dependerá de la pronta recuperación o deterioro del estado de salud del paciente durante su estancia en el hospital.

Evaluación: Se determina como la comparación planificada y sistematizada entre el estado de salud del paciente y los resultados esperados. Una vez finalizada la intervención de se evalúa a través del criterio de resultado NOC, contribuyendo a mejorar el nivel de conocimiento, desarrollo de habilidades y la motivación en el paciente para llevar a cabo y mantener acciones

\section{Intervención de enfermería en el cuidado del} paciente con diabetes mellitus

Cada una de las intervenciones permite tener conocimientos del paciente diabético sobre autocuidados, debido que a medida que avanza la edad, se toma conciencia del rol paciente y aumentan las probabilidades de progresar en el autocuidado. La taxonomía enfermera y el plan de cuidados en la historia clínica han permitido incrementar la comunicación interprofesional para mejorar la continuidad asistencial, a través de la mejora del Informe de Cuidados Enfermeros.

\section{Promoción de la salud, Nola Pender.}

Pender diseño el modelo de promoción de la salud, este modelo va dirigido a aumentar el nivel de bienestar del paciente, explicando la naturaleza multidimensional de las personas a la vez que interactúan dentro de su entorno para buscar bienestar y armonía. (Arrechedora, 2019)

Callista Roy, teoría de la adaptación. 
El modelo de adaptación de Roy contiene cinco elementos esenciales: Paciente, meta, salud, entorno y dirección de las actividades. Para planificar los cuidados propone un proceso de solución de problemas de seis pasos: Valorar la conducta del enfermo/usuario, valorar los factores influyentes, identificar los problemas, fijar los objetivos, seleccionar las intervenciones y evaluar los resultados. (Eduardo \& Leyla, 2017)

Marjory Gordon, patrones funcionales.

Marjory Gordon creó "los patrones funcionales de Gordon" una técnica de valoración para enfermería, esta guía servía para ayudar a los enfermeros a hacer un reconocimiento completo de sus pacientes; consiste en una lista de actividades y comportamientos que contribuyen a la salud y la calidad de vida de las personas. (Rodriguez, 2018).

\section{Teoría del autocuidado de Dorothea Orem.}

Describe su teoría como una contribución constante del individuo a su propia existencia, va dirigida hacia uno mismo, los demás y el entorno que nos rodea para regular los factores que afectan al desarrollo y funcionamiento en beneficio de la vida, salud o bienestar. Además, presenta esta teoría como una teoría general compuestas por tres teorías relacionadas entre sí: Teoría del autocuidado, teoría del déficit del autocuidado y teoría de los sistemas de enfermería. (laestenosisaortica's Blogs, 2016).

Teoría de Virginia Henderson sobre las 14 necesidades.

Es uno de los modelos de cuidados que mayor aprobación tiene en nuestro entorno. Elaborar un plan de cuidados individualizado para un paciente con Diabetes Mellitus se deben ofrecer unos cuidados de calidad y seguimiento en la consulta de atención primaria. La valoración de enfermería se puede realizar empleando el modelo de Virginia Henderson y aplicando las taxonomías enfermeras NANDA, NOC, NIC

\section{Diabetes Mellitus.}

La Diabetes Mellitus se refiere a una enfermedad metabólica crónica conocida por tener hiperglucemia, está relacionada con un déficit absoluto, pero también relativo de la producción o de la acción de la insulina.

Tipos de Diabetes Mellitus.

La diabetes mellitus se subdivide en tres categorías refiriéndose al mecanismo que provoca la hiperglucemia:

Diabetes Tipo 1: Conocida anteriormente diabetes mellitus insulinodependiente DMID o diabetes de inicio infanto-juvenil. Hay una insuficiencia absoluta de insulina. Se precisa insulina exógena para el debido control de la glucemia.

- Diabetes Tipo 2: Conocida como diabetes de inicio en la madurez: resistencia a la insulina con defectos variables en la destrucción. Suele asociarse a edades más avanzadas, frecuentemente a partir de los 40 años, pero también se diagnostica en niños y adolescentes con obesidad.

- Diabetes Gravídica o diabetes gestacional, de aparición en el embarazo (Bádenas, 2017).

Síntomas.

Los síntomas de la diabetes incluyen: Aumento de la sed y de las ganas de orinar, aumento del apetito, fatiga, visión borrosa, entumecimiento u hormigueo en las manos o los pies, úlceras que no cicatrizan y pérdida de peso sin razón aparente

Los síntomas de la diabetes tipo 1 pueden aparecer de manera espontánea y rápida, en cuestión hasta de semanas. En cambio, los síntomas pertenecientes a la diabetes tipo 2 pueden desarrollarse muy despacio, a lo largo de varios años, y pueden ser tan leves que a veces ni siquiera se notan ni mucho menos se sabe de su existencia. Muchas personas con diabetes tipo 2 no tienen síntomas. Algunas solo se enteran de que tienen la enfermedad cuando presentan problemas de salud relacionados con la diabetes, como visión borrosa o problemas del corazón.

\section{Causas}

No se conoce la causa exacta de la diabetes, entre otras cosas porque hay muchos tipos. De hecho, el momento de aparición de la enfermedad, las causas y los síntomas que presentan los pacientes dependen del tipo de diabetes:

-Diabetes tipo 1: Científicos piensan que la diabetes tipo 1 es causada por genes y factores ambientales, como los virus, que pueden desencadenar la enfermedad.

-Diabetes tipo 2: Hay mayor probabilidad de poseer diabetes tipo 2 si no se mantiene físicamente activa y tiene sobrepeso. En algunos casos, el sobrepeso provoca resistencia a la insulina y es frecuente en personas con diabetes tipo 2. El exceso de grasa en el vientre está relacionado con la resistencia a la insulina, la diabetes tipo 2, así como enfermedades del corazón y los vasos sanguíneos.

-Diabetes gestacional: Es causada por cambios hormonales, factores genéticos y factores del estilo de vida. Ciertas hormonas producidas por la placenta contribuyen a la resistencia a la insulina, que se dan en todas las mujeres hacia el final de la gestación. (Diseases, 2016)

\section{Tratamiento.}

Además de mantener un tratamiento farmacológico también es necesario de que el paciente mantenga una adecuada alimentación y en casos más graves llevar un plan de alimentación con el nutricionista, así como también ejercitarse. Un buen tratamiento de diabetes debe fundamentarse principalmente en el conocimiento de su fisiopatología. De modo que, la diabetes mellitus tipo 1 caracterizada por una 
insuficiencia de la secreción de la insulina y su único tratamiento hasta el momento es la administración de insulina, y en cuanto a la diabetes mellitus tipo 2 se puede administrar medicamentos orales.

\section{Factores de riesgo.}

En cuanto factores de riesgos muy importantes que amenazan la población con esta enfermedad son el consumo de comida grasa, azucares, y carbohidratos en excesos lo que con lleva a alterar los niveles de glucosa en la sangre, así como también otro factor muy influyente es el consumo excesivo de bebidas alcohólicas y llevar una vida sedentaria (Ivonne Roxanna Chong Delgado, 2019; Cristobal Franco Quinde, 2018).

Complicaciones.

Si el paciente no se controla o no mantiene un buen tratamiento, podría desarrollar complicaciones aguada e inclusive crónicas. Entre las complicaciones agudas, encontramos:

-Hipoglucemia: Es la complicación aguda de la diabetes y se define como un nivel de azúcar en la sangre menor de $70 \mathrm{mg} / \mathrm{dl}$. La causa es debido a un aporte insuficiente de hidratos de carbono en la comida, dosis de insulina excesiva, retrasos en la comida, demasiada actividad física y dosis excesiva de algunos hipoglucemiantes orales.

- Hiperglucemia severa: Situación metabólica en la que la cifra de hiperglucemia $>200 \mathrm{mg} / \mathrm{dl}$, puede desencadenar alteraciones más complejas a corto plazo como deshidratación, cetosis, etc.

-Cetosis: Presencia excesiva de cuerpos cetónicos en sangre, a causa del uso de las grasas como fuente de energía, puesto que falta insulina para utilizar la glucosa.

-Cetoacidosis: Situación extrema de una cetosis, con una reducción del $\mathrm{pH}$ de la sangre y requiere de tratamiento en un centro hospitalario.

Los síntomas se presentan en menos de 24 horas y comprenden: respiración de Kussmaul, aliento a cetonas, contracción del volumen del fluido extracelular, náuseas, vómitos y dolor abdominal, alteraciones en el nivel de consciencia (Dr. Gabriel Cuatrecasas, 2018; Gloriana Arroyo Sánchez, 2016)

- Hiperosmolar: Suele ocurrir en personas de mucha edad y el trastorno más importante es la deshidratación por hiperglucemia grave. Es una situación de alto riesgo vital que debe tratarse también en un centro hospitalario (Dr. Gabriel Cuatrecasas, 2018).

La alimentación como pilar fundamental para el control de la diabetes.

Mantener una alimentación adecuada es muy importante en la vida de los organismos vivos en los pacientes con diabetes es fundamental llevar un estilo de vida saludable es por ello que el artículo científico publicado por Polo de conocimiento indica que: consumir calorías de 20 a $25 \mathrm{Kcal}$ por $\mathrm{kg}$ de peso día, son suficientes para lograr y mantener un peso corporal saludable; carbohidratos no consumir $<130$ g/día, preferible los carbohidratos complejos reemplazar los simples por edulcorantes y distribuir de acuerdo al tratamiento farmacológico; grasas $<7$ de las calorías totales deben provenir de grasas saturadas y reducir grasas trans y colesterol a menos de $200 \mathrm{mg} /$ día. Proteínas 1.0 a $1.2 \mathrm{~g} / \mathrm{kg}$ de peso/día, adecuar según función renal y excreción de albúmina; fibra de 25 a 30 g/día y vitaminas minerales solo si sus necesidades fisiológicas lo ameritan (Ángel L. Zamora-Cevallos, 2018).

\section{Actividad física.}

La actividad física ayuda a mantener el nivel de glucosa, índices lipídicos y valores de presión arterial, también ayuda a mejorar aspectos asociados a la calidad de vida de individuos con DM2, demostrando efectos positivos sobre factores psicológicos, autoestima global y satisfacción con la vida. (Cascaes Silva, da Rosa Iop, \& Valdivia Arancibia, 2017).

Materiales y métodos

Dentro de esta sección se describe el diseño de investigación que se utilizó para obtener, recopilar la información necesaria y alcanzar el objetivo planteado. Se utilizó el método bibliográfico descriptivo porque permite realizar escritos sistemáticos y precisos, mostrando al investigador, el ¿cómo?, ¿por qué? y el ¿para qué? de una determinada situación. También se aplicó el método cualitativo ya que es el mejor método para recopilar datos concretos y descubrir detalles que permitan explicar por qué las personas llegan a sufrir esta dolencia.

Población y muestra: El estudio bibliográfico se llevó a cabo, trabajando con el total de la población que padece diabetes es más de 425 millones de personas a nivel mundial.

\section{Criterios de inclusión y exclusión}

Los criterios de inclusión son aquellas características que debe tener elementos de investigación (muestra), por ejemplo:

- Las cifras de pacientes que mueren por diabetes mellitus todos los días.

- Causas y riesgo que conlleva la diabetes mellitus, afectando a órganos, como los riñones y los ojos.

- Cuidados respectivos que se les debe brindar a los pacientes.

- Mayor riesgo de padecer diabetes mellitus en personas con sedentarismo, reduciendo la posibilidad de vida del paciente.

Por su parte, los criterios de exclusión son aquellos elementos de investigación que no presentan características muestrales, tales como: 
- Sabiendo que una de las causas de la diabetes mellitus es el sedentarismo, se excluyó a los deportistas durante el estudio.

- Pacientes con trastornos de enfermedad muy avanzados como insuficiencia renal terminal aquella dificulta el tratamiento y evolución en el paciente.

- Se excluye pacientes que deseen cumplir con su tratamiento.

\section{Resultados}

Los resultados obtenidos son en primera instancia, el conocimiento alcanzado referente a la Diabetes Mellitus, a los planes de cuidados y a los diversos procesos de atención de enfermería existentes y aplicados a nivel mundial. Otro de los resultados obtenidos fue la elaboración de una propuesta de un plan de cuidados.

Plan de cuidados para pacientes con Diabetes Mellitus.

\begin{tabular}{|c|c|c|c|}
\hline $\begin{array}{l}\text { Diagnostico } \\
\text { (NANDA) }\end{array}$ & $\begin{array}{l}\text { Resultados esperados } \\
\text { (NOC) }\end{array}$ & Intervenciones de enfermería (NIC) & Evaluación \\
\hline $\begin{array}{l}\text { Dominio 3: } \\
\text { Eliminación } \\
\text { Clase } \\
\text { Función } \\
\text { gastrointestinal } \\
\text { Estreñimiento } \\
\text { (00011) R/C } \\
\text { disminución de } \\
\text { la motilidad } \\
\text { del tracto } \\
\text { gastrointestinal } \\
\text { M/P con heces } \\
\text { formadas de } \\
\text { consistencia } \\
\text { dura color café } \\
\text { oscuro. }\end{array}$ & $\begin{array}{l}\text { Disminuir } \\
\text { distención } \\
\text { abdominal. mediante } \\
\text { la mayor ingesta de } \\
\text { fibra y } \\
\text { logrando líquidos, } \\
\text { evacuación } \\
\text { problemas ni dolor } \\
\text { alguno. } \\
\begin{array}{l}\text { En un lapso de un } \\
\text { mes. }\end{array}\end{array}$ & $\begin{array}{l}\text { Brindar información relacionado con la } \\
\text { ingesta de fibra y líquidos. } \\
\text {-Informarnos sobre la alimentación que } \\
\text { el paciente debe llevar. } \\
\text { Informar al paciente sobre la dieta que } \\
\text { va a llevar. } \\
\text {-Instruir al paciente sobre la } \\
\text { importancia de una dieta rica en fibras } \\
\text { y líquidos. } \\
\text { Manejo intestinal } \\
\text { - Controlar los movimientos } \\
\text { intestinales, incluyendo la frecuencia, } \\
\text { consistencia, forma, volumen y color } \\
\text { - Observar si hay signos y síntomas de } \\
\text { diarrea, estreñimiento o impactación. } \\
\text { Proporcionar apoyo emocional } \\
\text { - Aplicar el escucha activa } \\
\text { - Dialogar con el paciente } \\
\text { - Servir de enlace entre la familia y el } \\
\text { paciente }\end{array}$ & $\begin{array}{l}\text {-El paciente después } \\
\text { de realizadas todas las } \\
\text { intervenciones, logró } \\
\text { una evacuación sin } \\
\text { problema alguno sin } \\
\text { dolor. } \\
\text {-El paciente adquiere } \\
\text { conciencia sobre el } \\
\text { estilo de vida que } \\
\text { llevaba y lo mejora. }\end{array}$ \\
\hline
\end{tabular}




\begin{tabular}{|c|c|c|c|}
\hline $\begin{array}{l}\text { Dominio 2: } \\
\text { Nutrición } \\
\text { Clase 1: } \\
\text { Desequilibrio } \\
\text { nutricional por } \\
\text { exceso (0001) } \\
\text { R/C Déficit de } \\
\text { conocimiento, } \\
\text { aporte } \\
\text { excesivo de } \\
\text { nutrientes. }\end{array}$ & $\begin{array}{l}\text { Mantener un control } \\
\text { adecuado de peso y } \\
\text { brindarle al paciente } \\
\text { el } \\
\text { conocimiento } \\
\text { disponible de su } \\
\text { cuidado para así } \\
\text { lograr una mejoría } \\
\text { notoria de ru } \\
\text { condición } \\
\text { (DM). actual } \\
\end{array}$ & $\begin{array}{l}\text { Administración de una adecuada } \\
\text { nutrición } \\
\text { - Preguntar al paciente si tiene alergia a } \\
\text { algún alimento. } \\
\text { - Enseñar al paciente a llevar un diario } \\
\text { de comidas. } \\
\text { - Dar a conocer sobre los beneficios } \\
\text { que puede obtener siguiendo una } \\
\text { nutrición adecuado } \\
\text { Ayuda para disminuir el peso } \\
\text { - Motivar al paciente. } \\
\text { - Llevar un control sobre el peso del } \\
\text { paciente. } \\
\text { - Emplear un plan de cuidados } \\
\text { - Programar actividades de ejercicios } \\
\text { cada } 2 \text { a } 3 \text { veces en la semana. }\end{array}$ & $\begin{array}{l}\text {-Se ha logrado } \\
\text { visualizar en el } \\
\text { paciente que ha } \\
\text { mejorado } \\
\text { paulatinamente su } \\
\text { nutrición y su } \\
\text { condición física. } \\
\text {-Al aplicar todos estos } \\
\text { hábitos nutricionales } \\
\text { el paciente adquirió } \\
\text { un mejor estilo de vida } \\
\text { y mejoro su salud. }\end{array}$ \\
\hline $\begin{array}{l}\text { Dominio 2: } \\
\text { Nutrición } \\
\text { Clase 1: } \\
\text { Desequilibrio } \\
\text { nutricional por } \\
\text { exceso (0001) } \\
\text { R/C Déficit de } \\
\text { conocimiento, } \\
\text { aporte } \\
\text { excesivo de } \\
\text { nutrientes. }\end{array}$ & $\begin{array}{l}\text { Mantener un control } \\
\text { adecuado de peso y } \\
\text { brindarle al paciente } \\
\text { el mejor } \\
\text { conocimiento } \\
\text { disponible de su } \\
\text { cuidado para así } \\
\text { lograr una mejoría } \\
\text { notoria de su } \\
\text { condición } \\
\text { (DM). actual }\end{array}$ & $\begin{array}{l}\text { Administración de una adecuada } \\
\text { nutrición } \\
\text { - Preguntar al paciente si tiene alergia a } \\
\text { algún alimento. } \\
\text { - Enseñar al paciente a llevar un diario } \\
\text { de comidas. } \\
\text { - Dar a conocer sobre los beneficios } \\
\text { que puede obtener siguiendo una } \\
\text { nutrición adecuado } \\
\text { Ayuda para disminuir el peso } \\
\text { - Motivar al paciente. } \\
\text { - Llevar un control sobre el peso del } \\
\text { paciente. } \\
\text { - Emplear un plan de cuidados } \\
\text { - Programar actividades de ejercicios } \\
\text { cada } 2 \text { a } 3 \text { veces en la semana. }\end{array}$ & $\begin{array}{l}\text {-Se ha logrado } \\
\text { visualizar en el } \\
\text { paciente que ha } \\
\text { mejorado } \\
\text { paulatinamente su } \\
\text { nutrición y su } \\
\text { condición física. } \\
\text {-Al aplicar todos estos } \\
\text { hábitos nutricionales } \\
\text { el paciente adquirió } \\
\text { un mejor estilo de vida } \\
\text { y mejoro su salud. }\end{array}$ \\
\hline
\end{tabular}

Elaborado por autores del artículo

\section{Conclusiones}

Se concluye que:

- El rol de enfermero - paciente, es fundamental para llevar un cuidado correcto en el tratamiento de la Diabetes Mellitus, es aquí donde se atienden las necesidades del enfermo de una forma oportuna, segura y confiable.

- Por medio de la revisión bibliográfica se conoce tipos y procedimientos referentes al

- proceso de atención de enfermería aplicada a pacientes con diabetes mellitus. 

mortalidad por el incorrecto cuidado y control de la diabetes mellitus.

Colaboradores de la Investigación:

Álvarez Tituaña Sheyla Jamileth; Loor Cedeño Gema Jamileth; Rosales Cedeño Susana Noemí; Sornoza Rezabala Alexandra Monserrate; Tenelema Zambrano Miryan Monserrate; Tumbaco Saltos Helen Lissete; Veliz Ponce Sofía Daniela; Zambrano Coveña María Viviana; Zambrano Cevallos Sasha Nicolle.

\section{Bibliografía}

Ángel L. Zamora-Cevallos, W. J.-F.-L.-G.-L.-C. (2018). La importancia de la nutrición en pacientes diabéticos. Articulo cientifico, Polo de conocimiento, Ciencias de la salud, Manabí.

Arrechedora, I. (2019). Recuperado el 21 de Agosto de 2020, de Nola Pender: Biografía y Teoría.: https://www.lifeder.com/nola-pender/

Bádenas, D. J. (2017). Recuperado el 06 de Agosto de 2020, de Asociación Diabetes Madrid: https://diabetesmadrid.org/diabetes-tipo-1-tipo-2-definicion-diferencias/

Cascaes Silva, F., da Rosa lop, R., \& Valdivia Arancibia, B. A. (2017). Ejercicio físico, calidad de vida y salud de diabéticos tipo 2. Revista Psicologia del deporte , 26(1)

Cristobal Franco Quinde, E. N. (Febrero de 2018). Prevalencia y factores de riesgo de diabetes tipo II. Recimundo, 2(1).

Cynthia Colttersa, M. G. (11 de 2019). Gestion de cuidados de enfermeria en la persona mayor hospitalizada. Revista medica clinica de las Condes, 31(1).

Diseases, N. I. (2016). Recuperado el 06 de Agosto de 2020, de National Institute of Diabetes and Digestive and Kidney Diseases: https://www.niddk.nih.gov/health-information/informacion-de-lasalud/diabetes/informacion-general/sintomas-causas

Dr. Gabriel Cuatrecasas, D. J. (2018). Guia practica de las complicacones agudas de la diabetes. Guia practica, Amenarini diagnostic, Departamento de medicina interna, Barcelona.

Eduardo, B. V., \& Leyla, B. V. (2017). Afrontamiento y adaptación de los familiares desde el modelo de Callista Roy. Tesis, UNIVERSIDAD SEÑOR DE SIPÁN, Departamento de enfermería, Pimentel. Obtenido de http://repositorio.uss.edu.pe/bitstream/handle/uss/2997/TESIS-VILCHEZVILLEGAS\%20FINAL.pdf;sessionid=14F0B90AD98D1EDE1E5D830F083950E8? sequence=1

Enfermería, P. d. (2018). Recuperado el 12 de Agosto de 2020, de Ministerio de Salud Pública y Bienestar Social Instituto Nacional de Educación Permanente en Enfermería y Obstetricia: http://docs.bvsalud.org/biblioref/2018/11/964686/41-48.pdf

Gloriana Arroyo Sánchez, S. Q. (Enero de 2016). Cetoacidosis diabética y estado hiperglicémico hiperosmolar: un enfoque práctico. Revista Clínica de la Escuela de Medicina UCR - HSJD, 1(1).

Ivonne Roxanna Chong Delgado, H. M. (Septiembre de 2019). Diabetes mellitus, factores de riesgo y medidas preventivas en adolescentes del cantón Jipijapa. Recimundo, 3(3).

laestenosisaortica's Blogs. (2016). Recuperado el 22 de Agosto de 2020, de Teorias de Dorotea Orem (Autocuidado): https://laestenosisaortica.wordpress.com/teorias-de-dorotea-orem-autocuidado/

MSP. (2018). Recuperado el 5 de Agosto de 2020, de Ministerio de Salud Publica: https://www.salud.gob.ec/ministerio-de-salud-prevencion-y-autocuidado-son-claves-para-controlarla-diabetes/

Rodriguez, D. A. (2018). Recuperado el 22 de Agosto de 2020, de LIDEFER.COM: https://www.lifeder.com/marjory-gordon/ 